ITC $1 / 49$

Information Technology and Control

Vol. 49 / No. 1/ 2020

pp. 28-35

DOI /10.5755/j01.itc.49.1.23275
Improving Autonomous Performance of a Passive Morphing Fixed Wing UAV

\begin{tabular}{|l|l}
\hline Received 2019/05/19 & Accepted after revision 2019/12/17 \\
\hline
\end{tabular}

HOW TO CITE: Coban, S., Bilgic, H. H., \& Akan, E. (2020). Improving Autonomous Performance of a Passive Morphing Fixed Wing UAV. Information Technology and Control, 49(1), 28-35. https://doi.org//10.5755/j01.itc.49.1.23275

\title{
Improving Autonomous Performance of a Passive Morphing Fixed Wing UAV
}

\section{Sezer Coban}

Faculty of Aeronautics and Astronautics; Iskenderun Technical University; Iskenderun, Turkey; phone: +90 3266135600 (Ext: 5213); e-mail: sezer.coban@iste.edu.tr

\section{Hasan Huseyin Bilgic}

Faculty of Engineering and Natural Sciences; Iskenderun Technical University; Iskenderun, Turkey; phone: +90 3266135600 (Ext:2419); e-mail: hhuseyin.bilgic@iste.edu.tr

\section{Ercan Akan}

Barbaros Hayrettin Faculty of Naval Architecture and Maritime; Iskenderun Technical University; Iskenderun, Turkey; phone: +90 3266135600 (Ext: 3020); e-mail: ercan.akan@iste.edu.tr

Corresponding author: sezer.coban@iste.edu.tr

In this article, simultaneous longitudinal and lateral flight control systems design for both passive and active morphing unmanned aerial vehicles (UAVs) is first time applied for autonomous flight performance maximization. For this purpose, an UAV whose wing and tail unit can be assembled to fuselage from different points in a prescribed interval and whose wing and tail can move forward and backward independently in tail to nose direction is manufactured. Following this, an autopilot is purchased and it lets change of P, I, D coefficients in certain intervals. First, dynamic model and longitudinal and lateral state space models of UAV are obtained and then simulation model is reached. At the same time, block diagram of autopilot system and modeling of it in MATLAB/Simulink environment are found. After these, using these two models and adaptive stochastic optimization method, namely, SPSA, simultaneous design of UAV and autopilot is applied in order to minimize a cost function consisting of rise time, settling time and maximum overshoot. Therefore, primarily autonomous performance is maximized in computer environment. Moreover, high performance is observed at simulation responses.

KEYWORDS: UAV (Unmanned Aerial Vehicle), Dynamic Model, State Space Model, PID, Simulink. 


\section{Introduction}

Studies on the morphing of UAV have been increasing in recent years. The motivation behind morphing has been to find new ways to boost the capabilities of aircraft. This paper concentrates on UAV morphing concepts, designs and control technologies.

Aircraft wings are a structural part that allows the aircraft to fly in various flight conditions, but their performance can be improved. The ability to change the geometry of the wing surface before and during the flight attracts researchers and designers. Morphing means a change of shape, but there is no accepted definition in aviation. Changing the wing shape or geometry is not a new working area. In the literature, morphing solutions always have disadvantages in terms of cost, complexity or weight, but in some cases provide several benefits to the system. Recent developments in smart materials can overcome limitations and increase benefits from existing design solutions. The challenge is to design a structure that can withstand the prescribed loads, but can also change the shape of the structure. Ideally, there should be no distinction between the wing and the fuselage. Morphing is a promising technology for a new generation aircraft. However, manufacturers and end users still doubt about the benefits of adopting morphing in the near future. This paper provides a review of the latest technology in morphing aircraft and focuses on structural, deforming morphing concepts for fixed wings, particularly with reference to active systems. Researchers have carried out many studies on the modeling and control of unmanned aerial vehicles [8-15].

An Unmanned Aerial Vehicle (UAV) can move freely in three imaginary axes that intersect and are perpendicular to the center of gravity. To be able to say that this aircraft has been fully inspected, the pilot or automatic control systems must have control over the movement of these three imaginary axes. The Vertical Axis is that extending from the upper body through the center of gravity of the aircraft. Movement around this axis is called yaw motion. Yaw moves the nose of the aircraft to the right or left relative to the vertical axis. The longitudinal axis is the axis that extends from the nose to the tail of the aircraft, which crosses the aircraft and passes through the center of gravity. Motion around this axis is called 'roll'. The motion of the aircraft around this axis is controlled by flap, elevator or spoiler. The lateral axis is the axis that crosses the aircraft from one wing end to the other wing end and then passes through the center of gravity. Movement around this axis is called pitch motion. Pitch occurs when the aircraft changes its angle of attack, meaning that it moves to climb or dive [12]. The pitch movement of the aircraft around this axis is controlled by the elevators, movable horizontal stabilizers and elevators.

\section{UAV Designing and Manufacturing}

The design of UAV was completed on computer environment by using Solidworks program. A detailed drawing of all parts of the UAV during design was made. The top view of our unmanned aerial vehicle is given in Figure 1 [2].

Figure 1

Top view of UAV

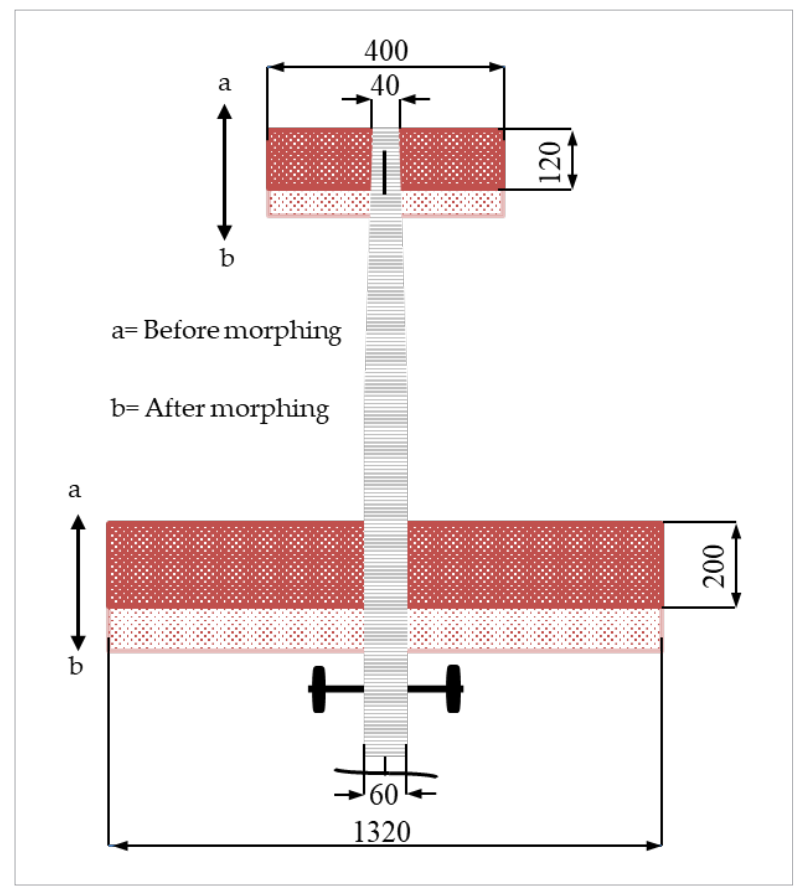

As a result of the designing, the parts of the body components were cut from the depron and the plywood in the laser cutting device. Body parts are glued together 
with epoxy adhesive. Wing and tail profile is produced using CNC cutting device. For the wing and tail set, the necessary reinforcements were made by using balsa, plywood and carbon rod. Aileron, elevator and rudder were produced. All outer surfaces were covered with coating. Transformation mechanism for wing and tail set was produced. Aileron, elevator and rudder were assembled. Front and rear landing gear were produced and assembled [4]. In Figure 2, UAV body and wing skeleton view and in Figure 3, the final version of the UAV are presented.

\section{Figure 2}

UAV body and wing skeleton view

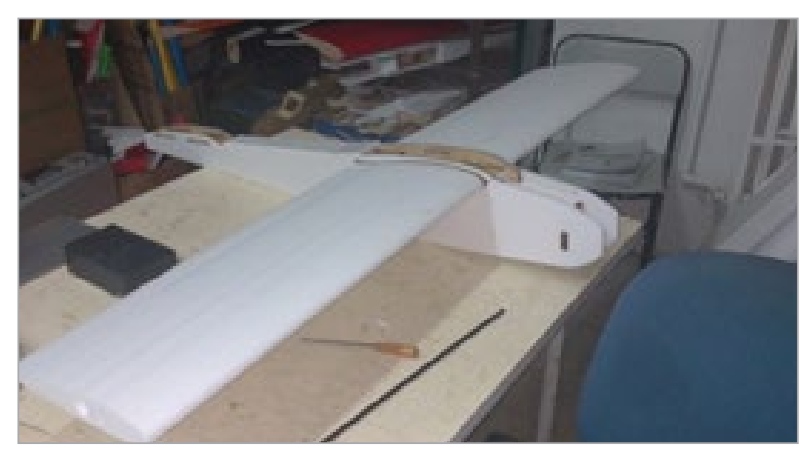

Figure 3

The final version of the UAV

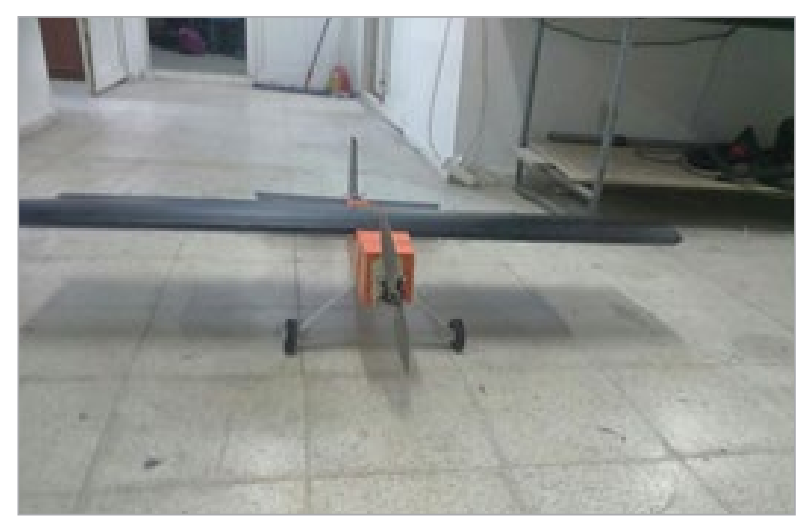

\section{UAV Dynamic Modeling}

In order to perform any dynamic modeling of any aeronautical vehicle or any UAV, it is necessary to obtain the governing equations of the aircraft body. These equations can be classified into three groups. These equations are the equations of force, moment equations and kinematic equations of the body. Newton's second law was used in the literature to extract the force equations [11]:

$$
{ }^{I} \vec{F}=M_{a} \frac{{ }^{I}}{d \vec{V}_{c g}} d=M_{a}\left[\frac{\partial \vec{V}_{c g}}{\partial t}+{ }^{I} \vec{\omega}^{A} \otimes \vec{V}_{c g}\right] .
$$

The components of the force on the three axes (X, Y, $\mathrm{Z})$ can be expressed in terms of the weight of the aircraft, linear accelerations, linear velocities $(u, v, w)$, angular velocities (p, q, r) and Euler orientation angles as follows [9]:

$$
F_{A}=\left[\begin{array}{c}
X \\
Y \\
Z
\end{array}\right]=\left[\begin{array}{c}
M_{a}(\dot{u}+q w-r v)+M_{a} g \sin \left(\theta_{A}\right) \\
M_{a}(\dot{v}+r u-p w)-M_{a} g \cos \left(\theta_{A}\right) \sin \left(\phi_{A}\right) \\
M_{a}(\dot{w}+p v-q u)-M_{a} g \cos \left(\theta_{A}\right) \sin \left(\phi_{A}\right)
\end{array}\right] .
$$

In order to extract the moment equations, the law of conservation of angular momentum, which is widely used in the literature, has been used. In Equation (3), this law is granted [14]:

$$
I \dot{\vec{h}}=\frac{\partial}{\partial t}^{I} \vec{h}+\vec{\omega}^{A} \otimes{ }^{I} \vec{h} .
$$

In Figure 4, the linear and angular velocity components in an aircraft (in the aircraft axis assembly) are presented visually. In Figure 5, the relationships between the speed components and the off-shore and side-strap angles are presented in an aircraft.

\section{Figure 4}

Linear and angular velocity components for aircraft (airborne axis team)

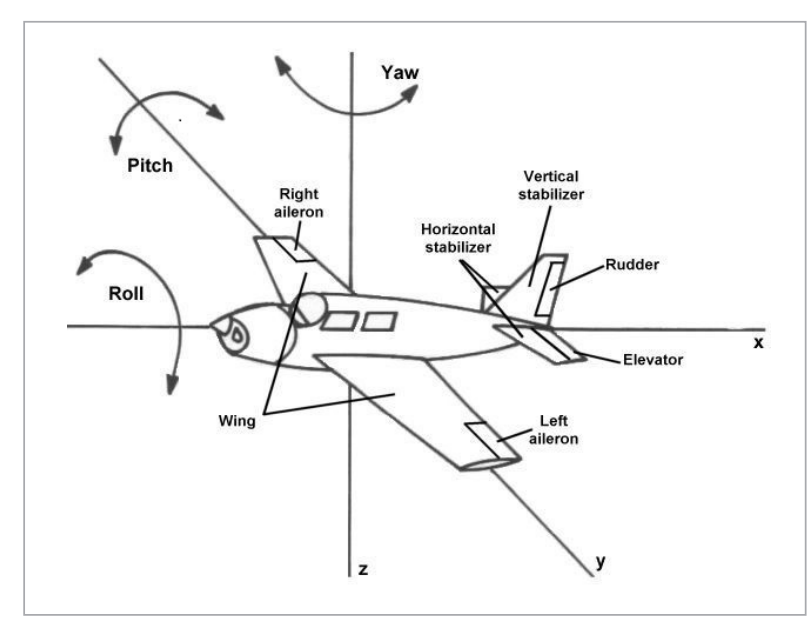




\section{Figure 5}

Ramp and side strap angles with speed components in an air tool

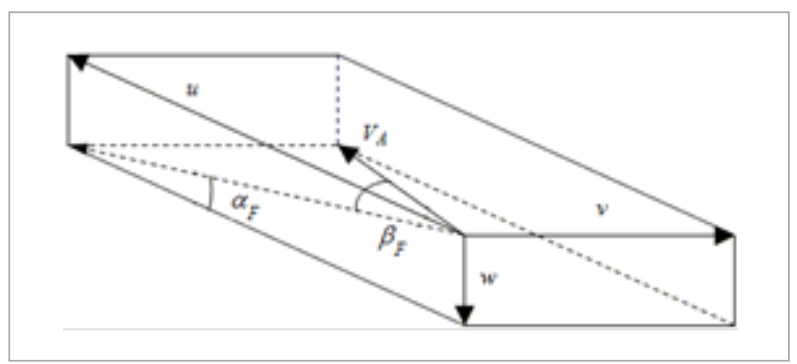

\section{State-Space Model}

Stability derivatives are obtained by using stability derivative coefficients. The state space model is derived using these obtained stability derivatives. Numerical values are obtained by using the geometric data of the unmanned aerial vehicle. Longitudinal state space model of UAV is given in Equation (4):

$$
\begin{aligned}
& {\left[\begin{array}{c}
\Delta \dot{u} \\
\Delta \dot{w} \\
\Delta \dot{q} \\
\Delta \dot{\theta}
\end{array}\right]=\left[\begin{array}{cccc}
X_{u} & X_{w} & 0 & -g \\
Z_{u} & Z_{w} & u_{0} & 0 \\
M_{u}+M_{\dot{w}} Z_{w} & M_{w}+M_{\dot{w}} Z_{w} & M_{q}+M_{\dot{w}} u_{0} & 0 \\
0 & 0 & 1 & 0
\end{array}\right]\left[\begin{array}{c}
\Delta u \\
\Delta w \\
\Delta q \\
\Delta \theta
\end{array}\right] \mathrm{L}} \\
& +\left[\begin{array}{cc}
X_{\delta_{T}} & X_{\delta_{e}} \\
Z_{\delta_{T}} & Z_{\delta_{e}} \\
M_{\delta_{T}}+M_{\dot{w}} Z_{\delta_{T}} & M_{\delta_{e}}+M_{\dot{w}} Z_{\delta_{e}} \\
0 & 0
\end{array}\right]\left[\begin{array}{l}
\Delta \delta_{T} \\
\Delta \delta_{e}
\end{array}\right]
\end{aligned}
$$

Lateral state space model is given in Equation (5) [16]:

$$
\begin{aligned}
& {\left[\begin{array}{c}
\Delta \dot{v} \\
\Delta \dot{p} \\
\Delta \dot{r} \\
\Delta \dot{\phi}
\end{array}\right]=\left[\begin{array}{cccc}
Y_{v} & Y_{p} & -\left(u_{0}-Y_{r}\right) & -g \cos \left(\theta_{0}\right) \\
L_{w}^{*}+\frac{I_{x z}}{I_{x}} N_{v}^{*} & L_{p}^{*}+\frac{I_{x z}}{I_{x}} N_{p}^{*} & L_{r}^{*}+\frac{I_{x z}}{I_{x}} N_{r}^{*} & 0 \\
N_{v}^{*}+\frac{I_{x z}}{I_{z}} L_{v}^{*} & N_{p}^{*}+\frac{I_{x z}}{I_{z}} L_{p}^{*} & N_{r}^{*}+\frac{I_{x z}}{I_{z}} L_{r}^{*} & 0 \\
0 & 1 & 0 & 0
\end{array}\right]\left[\begin{array}{c}
\Delta v \\
\Delta p \\
\Delta r \\
\Delta \phi
\end{array}\right]} \\
& +\left[\begin{array}{cc}
0 & Y_{\delta_{r}} \\
L_{\delta_{a}}^{*}+\frac{I_{x z}}{I_{x}} N_{\delta_{a}}^{*} & L_{\delta_{r}}^{*}+\frac{I_{x z}}{I_{x}} N_{\delta_{r}}^{*} \\
N_{\delta_{a}}^{*}+\frac{I_{x z}}{I_{z}} L_{\delta_{a}}^{*} & N_{\delta_{r}}^{*}+\frac{I_{x z}}{I_{z}} L_{\delta_{r}}^{*} \\
0 & 0
\end{array}\right]\left[\begin{array}{l}
\Delta \delta_{a} \\
\Delta \delta_{r}
\end{array}\right] .
\end{aligned}
$$

(5)
By examining the eigenvalues of state-space models obtained as a result of dynamic modeling, it can be decided that the models we create are correct. Figures 6 and 7 show the longitudinal and lateral flight modes, respectively. Therefore, it can be claimed that our dynamic modeling process is correct [6].

\section{Figure 6}

Longitudinal motion modes of UAV

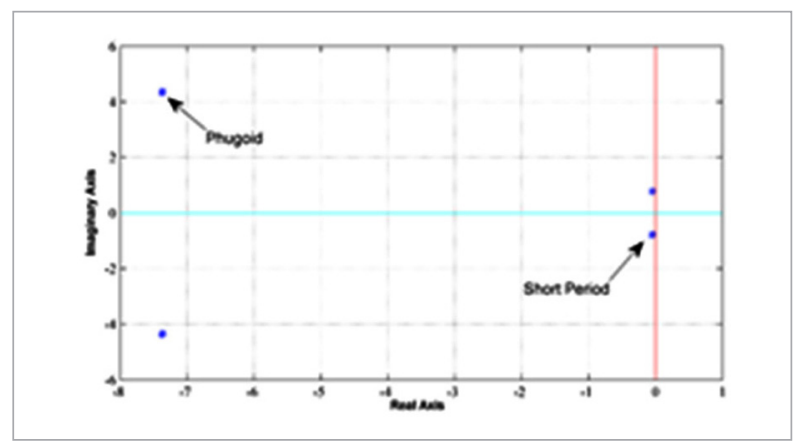

Figure 7

Lateral motion modes of UAV

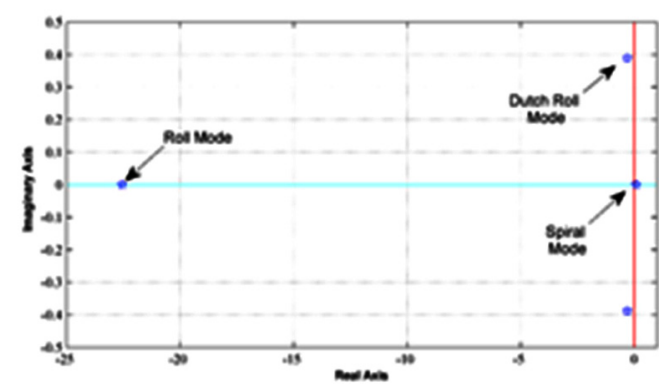

\section{Wing and Structural Analysis of UAV}

Figure 8 shows the aerodynamic coefficient values of UAV at different attack angles, such as $0^{\circ}, 4^{\circ}$ and $8^{\circ}$.

When we increase the wing profile at angles of $0^{\circ}$ to $20^{\circ}$ in angles of attack, the aerodynamic coefficients will change for each angle of attack. According to the results of the analysis, it is observed that the wing profile is stalled after $16^{\circ}$ of attack (Figure 9).

Figure 10(a) shows the deviation of the UAV on the wing. The deviation is increased as the wing moves towards the tip and reaches the highest value when the end of the wing is reached. Figure 10(b) shows the Von 


\section{Figure 8}

Aerodynamic coefficients at (a) $0^{\circ}$ attack angle, (b) $4^{\circ}$ attack angle, (c) $8^{\circ}$ attack angle

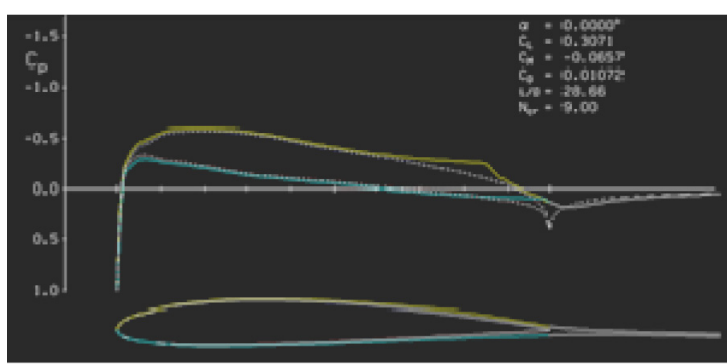

(a)

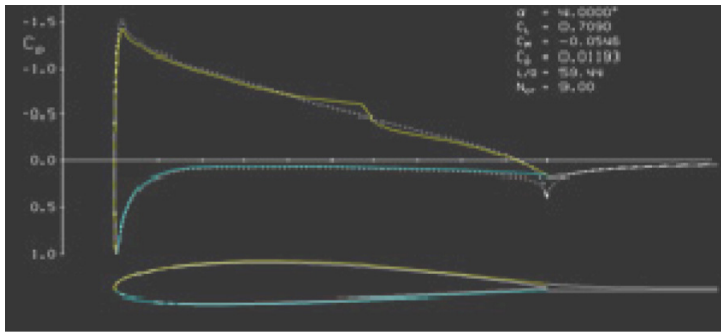

(b)

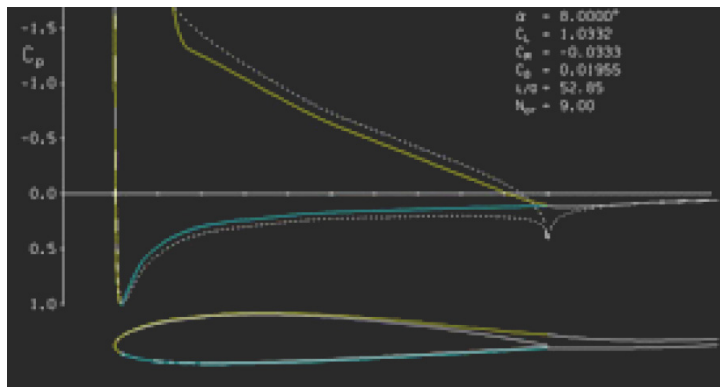

(c)

\section{Figure 9}

Angle of attack when entering the stall profile

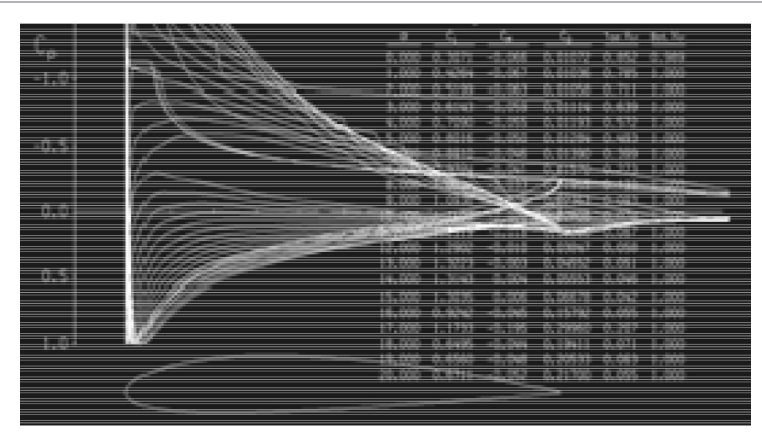

Mises stress values on the wing of the UAV. Von Mises stress values vary according to the change in bending resistance. As a result, the largest Von Mises strain value is found at the root of the wing. The best place for carbon tubes is where the voltage produced by the foam and carbon tubes is below the maximum voltage value.

\section{Figure 10}

Von mises stress results $(80 \mathrm{~km} / \mathrm{h})$

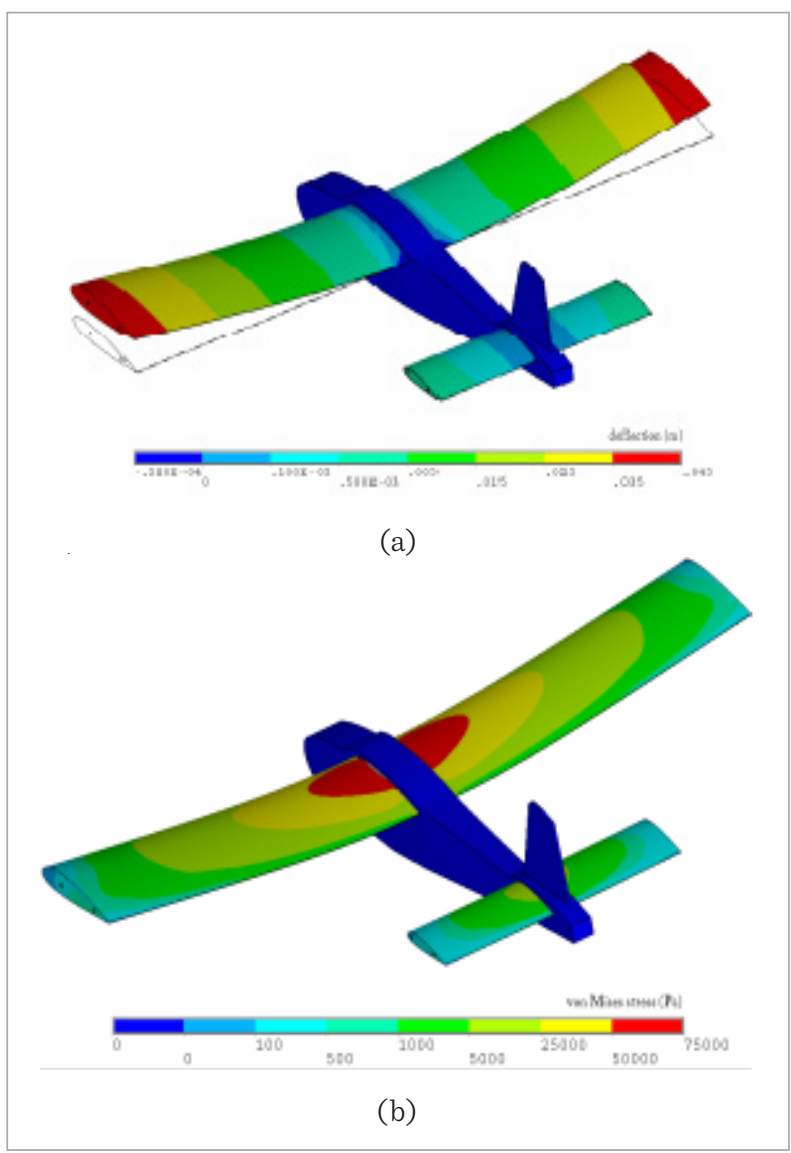

\section{Autopilot System and Optimization}

P-I-D based autopilot system is shown in Figure 11. Six P-I-D controllers were used to control the distance between the designated distances.

An adjustable autopilot was used for the flight observations. This autopilot system has a classical autopilot structure. The three layers for the hierarchical control structure are outer loop, middle loop and inner loop which have been shown in the Figure 12 [5]. Here, the trajectory analysis can be examined in 
Figure 11

Hierarchical detailed autopilot structure of the UAV

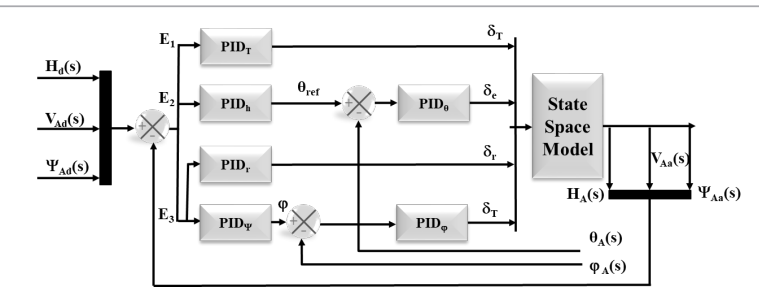

terms of speed, altitude or angle of yaw and their combination. It also benefited from 5 sensor inputs.

Streamlined strengths acting on an UAV body put ten-

\section{Figure 12}

The control structure of autopilot system

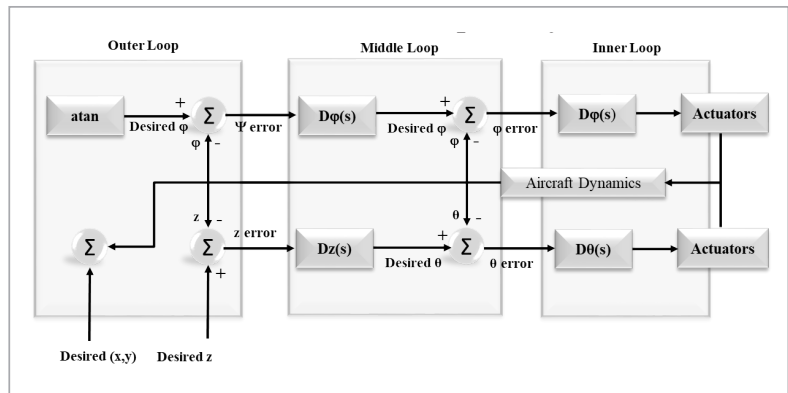

tatively within the wind burrow can be gotten with a drive estimation framework. In any case, it is very expensive to form the calculation of these forces by analyzing each body shape in an isolated wind burrow. In expansion, it is not possible to calculate nonlinear complex components logically contained within the streamlined strengths. For this reason, stochastic estimation strategies are utilized [3-10].

In complex problems like ours, where random gradient is not possible, random optimization methods should be used. SPSA and genetic algorithms are some of them. However, SPSA achieves optimal results much faster than genetic algorithms. Because SPSA performs two calculations at each iteration, genetic algorithms calculate up to $2 \mathrm{n}$. In other words, if the number of optimization variables is 5 , the SPSA performs 2 calculations in each iteration, while the genetic algorithms make 32 calculations. However, SPSA does not reach the optimum result every time that corresponding MATLAB code is executed. Therefore, a small number of trials is performed to determine the optimum result.
Since there is a complex dependence between total autonomous flight performance cost index and the constraints on the optimization variables (3 P-I-D gains for longitudinal controller, 3 P-I-D gains for lateral controller, and 2 passive morphing parameters of UAV and 2 active morphing parameters of UAV, entire of 10 parameters), computation of cost function derivatives with respect to these parameters is not analytically possible. This advocates the invitation of certain stochastic optimization techniques. In order to solve this specific problem, a stochastic optimization method, named as SPSA (i.e. simultaneous perturbation stochastic approximation), is applied.

\section{Results and Discussion}

As a result of the concurrent design, a significant improvement was achieved in the performance index consisting of settling time, rising time and maximum overshoot. This ratio is $59 \%$ and is obtained from the relative relationship between the value obtained without concurrent calculation and the results obtained from the concurrent calculation.

\section{Figure 13}

(a) Cost improvement, (b) Relative cost improvement of the longitudinal motion

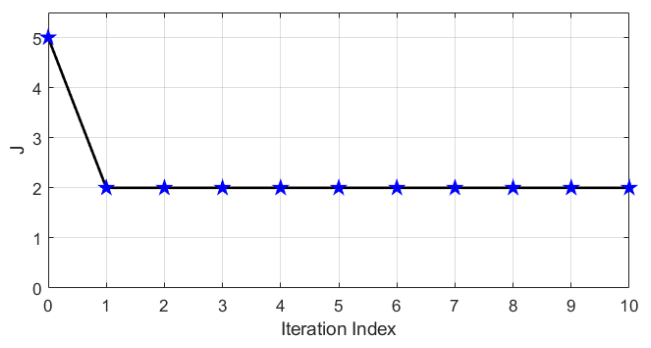

(a)

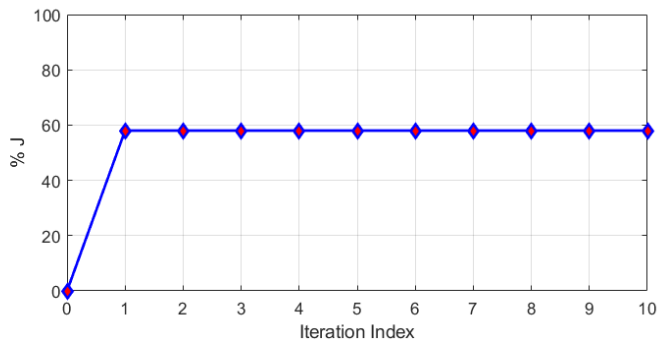

(b) 
Figure 14

Closed-loop responses of longitudinal motion
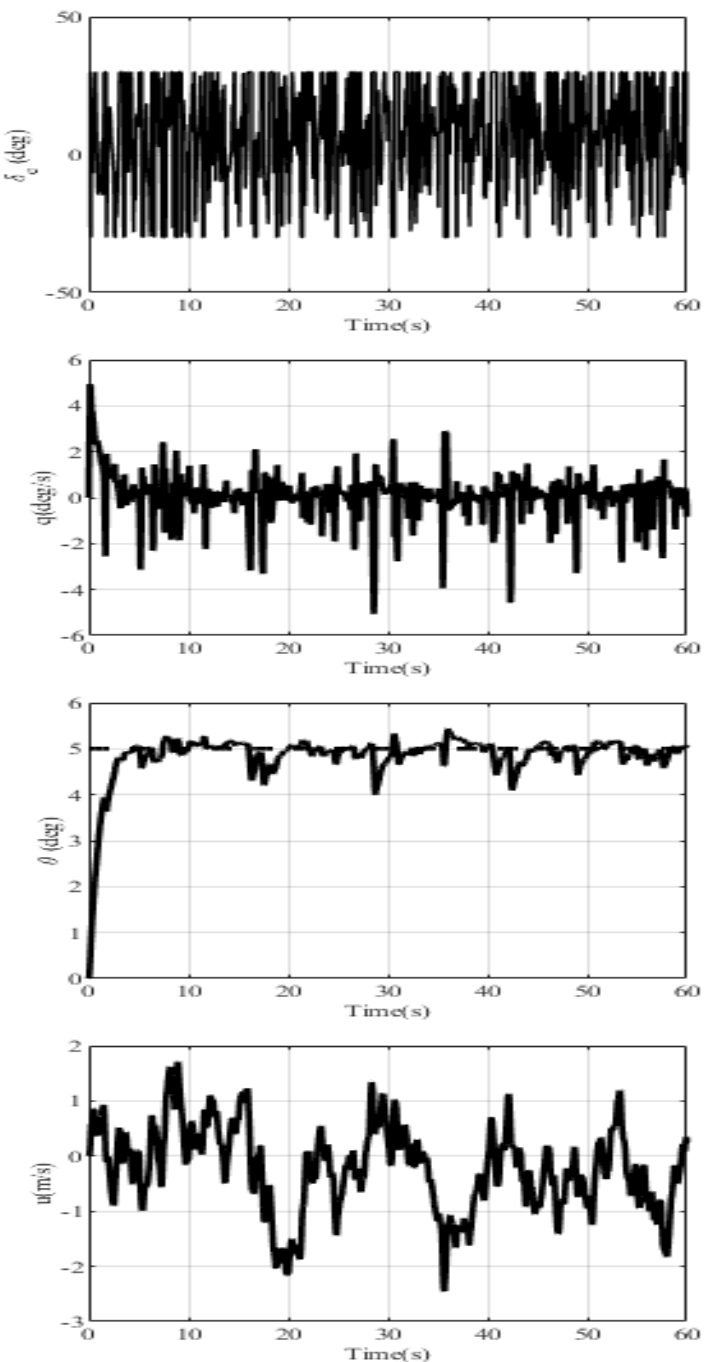

Figure 14 shows the pitch angle of the longitudinal motion, the velocity along the x-axis, the angular velocity along the y-axis, the velocity along the z-axis and the responses of the horizontal tail and the elevation angle.

\section{References}

1. AL-Madani, B., Svirskis, M., Narvydas, G., Maskeliūnas, R., Damaševičius, R. Design of Fully Automatic Drone Parachute System with Temperature Compensation
As can be seen from Figure 14, the longitudinal trajectory has followed the pitch angle well with a little error of 3 degrees. Furthermore, the velocity along the $\mathrm{x}$-axis, the angular velocity along the $\mathrm{y}$-axis followed by the trajectory zero line.

\section{Conclusions}

In this paper, simultaneous longitudinal and lateral flight control systems design for both passive and active morphing unmanned aerial vehicles (UAVs) is first time applied for autonomous flight performance maximization. Dynamic model and longitudinal and lateral state space models of UAV are obtained and then simulation model of UAV is reached. After these, adaptive stochastic optimization method (SPSA), simultaneous design of UAV and autopilot is applied in order to minimize a cost function consisting of rise time, settling time and maximum overshoot. As a result of the concurrent design, a significant improvement was achieved in the performance index consisting of settling time, rising time and maximum overshoot and this ratio is $59 \%$. Since the total cost index captures terms both related with longitudinal and lateral flights, considerable improvement in longitudinal autonomous flight performance was obtained and the lateral autonomous flight performance were not broken. Closed loop responses for both longitudinal and lateral flight while there exist atmospheric turbulence were investigated. The desired trajectories (i.e. 3 degrees roll angle for lateral autopilot and 3 degrees pitch angle for longitudinal autopilot) were successfully tracked. The saturations on active control surfaces (i.e. elevator and aileron) were also satisfied. In addition, the other outputs such as linear and angular velocities were not experienced with catastrophic behavior. Simultaneous design idea converted the UAV and its autopilot system into suitable form satisfying good performance and trajectory tracking for both lateral and longitudinal flights.

Mechanism for Civilian and Military Applications. Journal of Advanced Transportation, 2018, 1-11. https:// doi.org/10.1155/2018/2964583 
2. Antić, D., Jovanović, Z., Danković, N., Spasić, M., Stankov, S. Probability Estimation of Certain Properties of the Imperfect Systems. Proceedings of IEEE 7th International Symposium on Applied Computational Intelligence and Informatics, (SACI 2012), Timisoara, Romania, May 24-26, 2012, 213-216. https://doi.org/10.1109/ SACI.2012.6250004

3. Bilgic, H. H., Sen, M. A., Kalyoncu, M. Tuning of LQR Controller for an Experimental Inverted Pendulum System Based on the Bees Algorithm. Journal of Vibroengineering, 2016, 18(6), 3684-3694. https://doi. org/10.21595/jve.2016.16787

4. Brodić, D. Advantages of the Extended Water Flow Algorithm for Handwritten Text Segmentation. In: Kuznetsov, S. O. et al. (Eds.), Pattern Recognition and Machine Intelligence, Lecture Notes in Computer Science, 6744, Springer, Belin-Heidelberg, 2011, 418-423. https://doi.org/10.1007/978-3-642-21786-9_68

5. Çoban S., Oktay T. A Review of Tactical Unmanned Aerial Vehicle Design Studies; The Eurasia Proceedings of Science, Technology, Engineering Mathematics; 2017, (1), 30-35.

6. Çoban S., Oktay T. Legal and Ethical Issues of Unmanned Aerial Vehicles. Journal of Aviation, 2018, 2(1), 31-35. https://doi.org/10.30518/jav.421644

7. Elbanna, A. E. A., Soliman, T. H. M., Ouda, A. N., Hamed, E. M. Improved Design and Implementation of Automatic Flight Control System (AFCS) for a Fixed Wing Small UAV. Radioengineering, 2018, 27(3), 882-890. https://doi.org/10.13164/re.2018.0882

8. Gandolfo, D. C., Salinas, L. R., Brandão, A. S., Toibero, J. M. Path Following for Unmanned Helicopter: An Approach on Energy Autonomy Improvement. Infor- mation Technology and Control, 2016, 45(1), 86-98. https://doi.org/10.5755/j01.itc.45.1.12413

9. Ivanovas, A., Ostreika, A., Maskeliūnas, R., Damaševičius, R., Połap, D., Woźniak, M. Block Matching Based Obstacle Avoidance for Unmanned Aerial Vehicle. Lecture Notes in Computer Science, 2018, 58-69. https://doi. org/10.1007/978-3-319-91253-0_6

10. Oktay T., Çoban, S. Simultaneous Longitudinal and Lateral Flight Control Systems Design For both Passive and Active Morphing Tuavs. Elektronika Ir Elektrotechnika, 2017, 23(5), 15-20. https://doi.org/10.5755/ j01.eie.23.5.19238

11. Preparata, F. P., Shamos, M. I. Computational Geometry: An Introduction. Springer, Berlin, 1995.

12. Spall, J. C. Multivariate Stochastic Approximation Using A Simultaneous Perturbation Gradient Approximation. IEEE Transactions on Automatic Control, 1992, 37(3), 332-341. https://doi.org/10.1109/9.119632

13. Tsai, J.L.EfficientMulti-Server Authentication Scheme Based on One-Way Hash Function Without Verification Table. Computers and Security, 2008, 27(3), 115-121. https://doi.org/10.1016/j.cose.2008.04.001

14. Vidojković, B., Jovanović, Z., Milojković, M. The Probability Stability Estimation of the System Based on the Quality of the Components. Facta Universitatis, Series: Electronics and Energetics, 2006, 19(3), 385-391. https://doi.org/10.2298/FUEE0603385V

15. Yazid, E., Garratt, M., Santoso, F. Control Position of A Quadcopter Drone Using Evolutionary Algorithms Optimized Self-Tuning 1st-Order Takagi-Sugeno-Kang-Type Fuzzy Logic Controller. Applied Soft Computing, 2019, (1)78, 373-39. https://doi. org/10.1016/j.asoc.2019.02.023 